\title{
Immunohistochemical differential diagnosis between thymic carcinoma and type B3 thymoma: diagnostic utility of hypoxic marker, GLUT-1, in thymic epithelial neoplasms
}

\author{
Masakazu Kojika ${ }^{1,2}$, Genichiro Ishii ${ }^{1}$, Junji Yoshida ${ }^{2}$, Mituyo Nishimura ${ }^{2}$, \\ Tomoyuki Hishida ${ }^{2}$, Shu-ji Ota ${ }^{1}$, Yukinori Murata ${ }^{1}$, Kanji Nagai ${ }^{2}$ and Atsushi Ochiai ${ }^{1}$ \\ ${ }^{1}$ Pathology Division, Research Center for Innovative Oncology, National Cancer Center Hospital East, \\ Kashiwa, Chiba, Japan and ${ }^{2}$ Thoracic Surgery Division, National Cancer Center Hospital East, Kashiwa, \\ Chiba, Japan
}

\begin{abstract}
There are only a few immunohistochemical markers that are useful for differentiating thymic carcinomas from type B3 thymomas. The purpose of this study is to examine the additional markers that would be useful for differentiating between thymic carcinoma and thymoma type B3. We performed a tissue microarray analysis of surgically resected thymic tumor specimens from 12 cases of thymic carcinoma, 7 cases of type B3 thymoma, and 68 cases of other types of thymoma. Immunostaining using 49 antibodies was scored based on staining intensity and the percentage of cells that stained positive. Seven proteins that were selected by the staining scores, namely, GLUT-1 (167 vs 4), CA-IX (110 vs 15), C-kit (162 vs 44), CD5 (33 vs 0), MUC-1 (54 vs 0), CEA (42 vs 0), and CK18 (110 vs 42), were significantly higher in the thymic carcinomas than in the type B3 thymomas. The staining sensitivity and specificity of the antibodies for thymic carcinoma were GLUT-1, sensitivity $72 \%$ and specificity 100\%; CA-IX, 58 and 71\%; C-kit, 72 and 85\%; CD5, 33 and 100\%; CK18, 58 and 71\%; MUC-1, 25 and $100 \%$; and CEA, 33 and $100 \%$. Glucose transporter 1 (GLUT-1) is the best marker for thymic carcinoma because it had the highest sensitivity and specificity. Positive immunostaining for a combination of three markers, namely, GLUT-1, CD5, and CEA, enabled differentiation of thymic carcinoma with $91.6 \%$ sensitivity and $100 \%$ specificity. In conclusion, we identified GLUT-1 as an additional marker that will be useful for differentiating thymic carcinoma from type B3 thymoma, especially in biopsy specimens that have been crushed or are otherwise difficult to examine morphologically in thymic tumors.
\end{abstract}

Modern Pathology (2009) 22, 1341-1350; doi:10.1038/modpathol.2009.105; published online 31 July 2009

Keywords: thymic carcinoma; thymoma; type B3 thymoma; GLUT-1; CA-IV

The thymus is an immune-related organ, whose network-forming epithelial cells are responsible for immature T-cell (thymocyte) growth, development, and differentiation. Thymic epithelial tumors are traditionally classified as thymomas and thymic carcinomas. Thymomas consist of neoplastic epithelial cells and immature $\mathrm{T}$ cells intermingled in variable proportions, whereas thymic carcinomas

Correspondence: Dr A Ochiai, MD, PhD, Pathology Division, Research Center for Innovative Oncology, National Cancer Center Hospital East, 6-5-1, Kashiwanoha, Kashiwa-City, Chiba 277-8577, Japan.

E-mail: aochiai@east.ncc.go.jp

Received 16 February 2009; revised and accepted 2 June 2009; published online 31 July 2009 consist of tumor cells similar to carcinomas occurring in other organs with infiltrating mature $\mathrm{T}$ cells. However, little is known about the pathogenesis of thymoma or thymic carcinomas.

In 1999, the World Health Organization (WHO) published a classification of thymic epithelial tumors that is based on the morphology of the epithelial cells and the lymphocyte-to-epithelial cell ratio of the tumor. Thymic epithelial tumors are classified into five types: A, AB, B1, B2, and B3. In type A the epithelial cells appear spindle-shaped or oval, whereas in type B they are dendritic or plump, and tumors that combine these two morphologies are designated as type AB. Type B thymomas are subdivided into subtypes B1, B2, and B3, increasing 
with the ratio of atypia in the neoplastic cells. Type B3 thymoma was predominantly epithelial type, with severe cellular atypia and a few immature $\mathrm{T}$ cells around the tumor cells, and was considered to be a borderline malignancy. On the other hand, all thymic carcinomas are classified as type $\mathrm{C}$ thymomas. In 2004, the WHO classification of thymic tumors was revised and the term 'type C thymoma' was replaced by 'thymic carcinoma.' ${ }^{1,2}$ The validity of this classification as a prognostic tool is a matter of controversy, even if the preliminary studies are encouraging. It is sometimes difficult to make the differential diagnosis between thymoma type B3 and thymic carcinoma histologically, especially when the biopsy specimen is small. Although immunoreactivity for CD5 and c-kit has been reported as a useful marker for primary thymic carcinoma, but not for thymoma, the positive rate of CD5 and c-kit has been reported to be limited to over $50 \% ;^{3-7}$ and as not all neoplastic cells in thymic carcinomas stain positive for these markers, ${ }^{8-11}$ it will be necessary to identify other diagnostic markers to make the differential diagnosis more reliable.

In this study, we used a tissue microarray with a large panel of antibodies to identify additional immunohistochemical markers to phenotypically differentiate between thymic carcinomas and type B3 thymomas.

\section{Materials and methods}

\section{Case Selection}

During the period from January 1992 to December 2006, a total of 94 patients with thymic epithelial tumors were treated at the National Cancer Center Hospital East, Chiba, Japan. All thymic epithelial tumors, with a pathological diagnosis based on the classification schema of the third edition of the WHO classification, were reviewed, and 18 tumors were diagnosed as type A thymoma, 27 as type B1 and 10 as type $\mathrm{B} 2$ thymoma, 12 as type $\mathrm{AB}$ thymoma, and 18 as thymic carcinoma. The six cases of thymic carcinoma, and four cases of thymoma, for which an adequate tissue specimen was not available for pathological review, were excluded from the study, leaving a total of 84 cases of thymic epithelial tumor for review. The tumors selected for tissue microarray analysis were: 10 cases of type A thymoma, 5 cases of type B1 thymoma, 5 cases of type B2 thymoma, 7 cases of type B3 thymoma, and 12 cases of thymic carcinomas. The 12 cases of thymic carcinoma consisted of 6 cases of keratinizing carcinoma and 6 cases non-keratinizing carcinoma. Representative figures of type B3 thymoma and thymic carcinoma are shown in Figure 1a, b, c, and d.

\section{Pathological Studies}

After fixing the specimens with $10 \%$ formalin and embedding them in paraffin, serial $4-\mu \mathrm{m}$ sections were stained using hematoxylin and eosin (H\&E). The sections were reviewed by two pathologists (MK, GI), according to the histological criteria described in the WHO classification, and the discrepancies were resolved by joint discussion of the slides while viewing with a multiheaded microscope. In addition to the morphological examination, we examined the sections for the presence of immature $\mathrm{T}$ thymocyte using CD99 and terminal deoxynucleotidyl transferase immunostaining.

\section{Construction of Tumor Tissue Microarrays}

Tissue microarrays were constructed according to the procedure described in previous reports. ${ }^{12-14}$ Briefly, the most representative tumor areas were carefully selected and marked on the H\&E-stained slide to construct the microarrays, and the tissue microarrays were assembled using a tissue arraying instrument (KIN-1; Azumaya, Tokyo, Japan). ${ }^{15}$ The microarray system consists of thin-walled stainless steel needles approximately $2 \mathrm{~mm}$ in diameter, and a stylet for transferring and removing the contents of the needle. The assembly is held in an $\mathrm{x}-\mathrm{y}$ position guide that is manually adjusted using digital micrometers. Core samples are retrieved from selected areas of donor tissue and precisely arrayed in a new (recipient) paraffin block. Samples of the specimens were routinely obtained by collecting two replicate core samples of the tumor from different areas. The normal thymus tissue and liver tissue, used as the positive/negative control, were arrayed in sample paraffin blocks.

\section{Antibodies and Immunohistochemical Staining}

The 49 antibodies used in this study are listed in Table 1. Immunohistochemical staining was performed according to the procedure described in a previous report. ${ }^{13}$ Tissue microarray donor blocks were cut into $4-\mu \mathrm{m}$ sections and mounted on silanecoated slides. The sections were then deparaffinized in xylene and dehydrated in a graded alcohol series, and the endogenous peroxidase was blocked using $3 \%$ hydrogen peroxide in absolute methyl alcohol. Heat-induced epitope retrieval was performed for 20 minutes at $95^{\circ} \mathrm{C}$ using a $0.02-\mathrm{mol} / \mathrm{l}$ concentration of citrate buffer ( $\mathrm{pH}$ 6.0). After allowing the slides to cool at room temperature for 60 minutes, they were rinsed with deionized water and incubated overnight with the primary antibodies. The slides were then washed thrice with phosphate-buffered saline and incubated with the EnVision + System-HRP (Dako, Glostrup, Denmark). The reaction products were stained using diaminobenzidine and counterstained using hematoxylin. Some antibodies (Table 1) were used in an automated immunostainer (Ventana Medical Systems, Tucson, AZ, USA) after antigen retrieval by microwave heating and using citrate buffer. Immunostaining was recorded as 

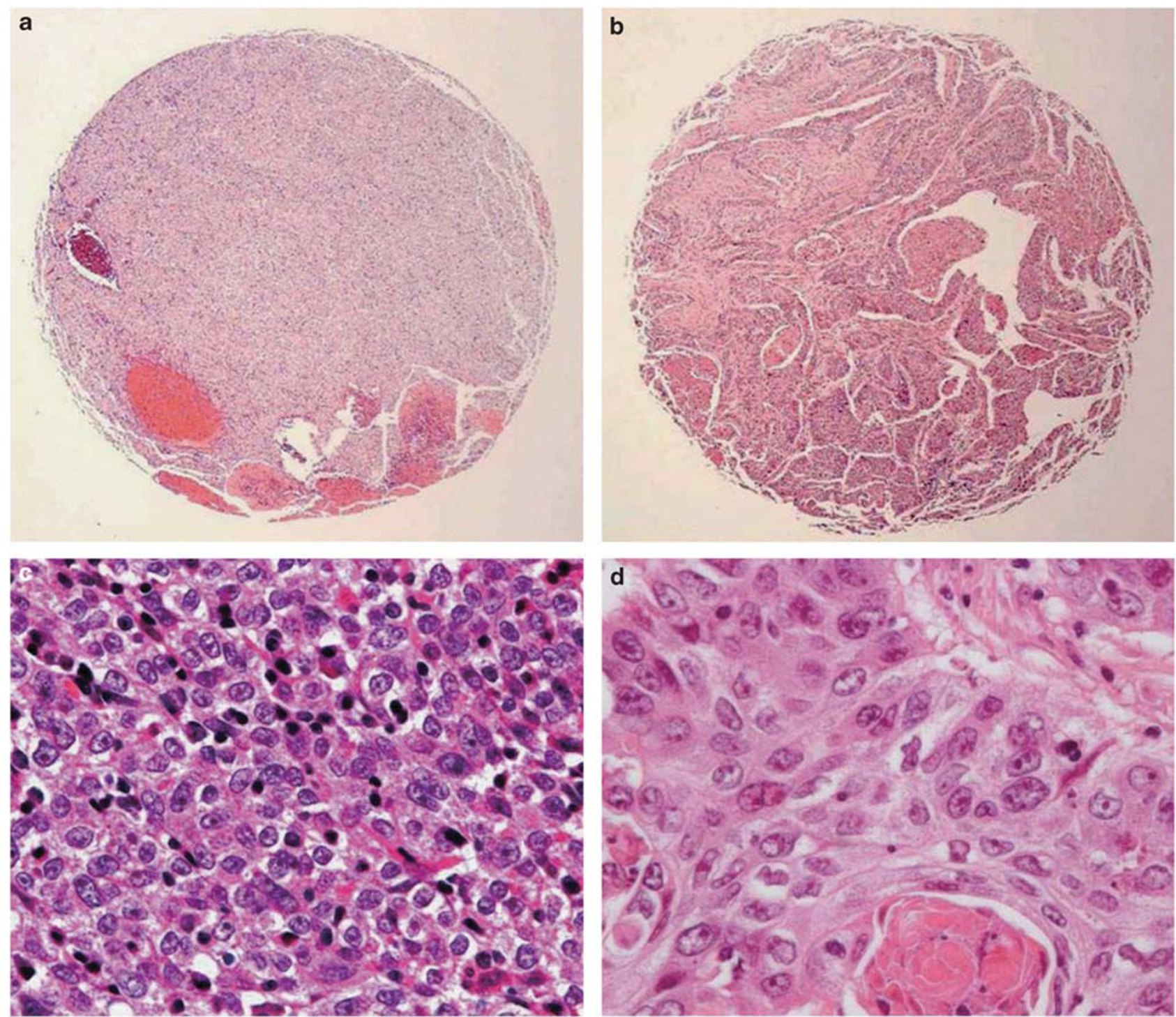

Figure 1 Preparation of tissue microarray using hematoxylin and eosin stain. (a) Histological features of type B3 thymoma. (b) Histological features of thymic carcinoma. (c) Histological features of type B3 thymoma (higher magnification). Severe cellular atypia with conspicuous nucleoli and a few immature T cells around the tumor cells are seen. (d) Histological features of thymic carcinoma (higher magnification).

positive when more than $20 \%$ of the cancer cells exhibited intermediate or strong staining (staining intensity $=2,3)$.

\section{Immunohistochemical Scoring and Criteria for Positive Staining}

The tissue sections were semiquantitatively scored separately for membranous and cytoplasmic staining by light microscopy. Staining scores were calculated by multiplying the percentage of positive tumor cells per slide $(0-100 \%)$ by the predominant level of staining intensity: $0=$ negative, $1=$ weak, $2=$ intermediate, and $3=$ strong. Possible scores ranged from 0 to 300 . The scores of two stained samples were averaged, and the result was recorded as score for that tumor. The staining scores obtained for the two samples from the same specimen were calculated. If one sample was lost, the staining score was calculated from the data for the remaining sample alone.

\section{Statistical Analysis}

The prism statistical software package (GraphPad Software, San Diego, CA, USA) was used to perform the statistical analyses. The correlations between the thymic carcinoma group and the thymoma group, and the results of immunohistochemical staining were evaluated by the $\chi^{2}$-test or Fisher's exact test, as appropriate. $P$-values $<0.05$ were considered significant. 
Table 1 Used antibodies list

\begin{tabular}{|c|c|c|c|c|}
\hline Classification/antibody & Clone & Pretreatment & Dilution & Source \\
\hline \multicolumn{5}{|l|}{ Cytokeratins } \\
\hline CK1 & $34 \beta \mathrm{B} 4$ & Microwave & $1: 20$ & Novocastra, Newcastle upon Tyne, England \\
\hline CK4 & $6 \mathrm{~B} 10$ & Microwave & $1: 100$ & Novocastra \\
\hline CK5/6 & $\mathrm{D} 5 / 16 \mathrm{~B} 4$ & Microwave & $1: 50$ & Dako Cytomation, Carpinteria, CA, USA \\
\hline CK7 & OV-TL 12/30 & Microwave & $1: 50$ & Dako Cytomation \\
\hline CK8 & TS1 & Microwave & $1: 50$ & Novocastra \\
\hline CK10 & LHP1 & Microwave & $1: 50$ & Novocastra \\
\hline CK13 & $\mathrm{KS}-1 \mathrm{~A} 3$ & Microwave & $1: 100$ & Novocastra \\
\hline CK14 & CKB1 & Microwave & $1: 20$ & Sigma-Aldrich, St Louis, MO, USA \\
\hline CK15 & LHK15 & Microwave & $1: 40$ & Novocastra \\
\hline CK17 & E3 & Microwave & $1: 20$ & Dako Cytomation \\
\hline CK18 & DC10 & Microwave & $1: 25$ & Dako Cytomation \\
\hline CK19 & RCK108 & Microwave & $1: 50$ & Dako Cytomation \\
\hline CK20 & Ks20.8 & Microwave & $1: 25$ & Dako Cytomation \\
\hline \multicolumn{5}{|c|}{ Cytoskeletal-associated markers } \\
\hline S-100 & Polyclonal & Microwave & Prediluted & Ventana Medical Systems, Tucson, AZ, USA \\
\hline Vimentin & $3 \mathrm{~B} 4$ & Microwave & Prediluted & Ventana Medical Systems \\
\hline \multicolumn{5}{|c|}{ Drug-resistant gene products } \\
\hline ERCC-1 & $8 \mathrm{~F} 1$ & Autoclave & $1: 200$ & Labvision, CA, USA \\
\hline \multicolumn{5}{|c|}{ Apoptosis-associated proteins } \\
\hline bcl-2 & 124 & Microwave & $1: 40$ & Dako Cytomation \\
\hline P53 & Polyclonal & Microwave & $1: 1000$ & Nichirei Biosciences, Japan \\
\hline P63 & $\mathrm{A} 4 \mathrm{~A}$ & Microwave & $1: 200$ & Dako Cytomation \\
\hline \multicolumn{5}{|c|}{ Growth factors and hormone receptors } \\
\hline EGFR & 113 & Microwave & $1: 10$ & Novocastra \\
\hline c-erbB-2 & CB11 & Microwave & Prediluted & Ventana Medical Systems \\
\hline IGFR & $24-31$ & Microwave & $1: 100$ & Chemicon, Temecula, CA, USA \\
\hline c-kit & Polyclonal & Microwave & $1: 50$ & Dako Cytomation \\
\hline PgR & $1 \mathrm{~A} 6$ & Microwave & Prediluted & Ventana Medical Systems \\
\hline $\mathrm{ER}$ & $6 \mathrm{~F} 11$ & Microwave & Prediluted & Ventana Medical Systems \\
\hline AR & AR441 & Microwave & $1: 50$ & Dako Cytomation \\
\hline $\operatorname{PDGFR} \beta$ & PR7212 & Microwave & $1: 100$ & R\&D System, Minneapolis, MN, USA \\
\hline \multicolumn{5}{|l|}{ Adhesion molecules } \\
\hline$\beta$-catenin & 14 & Microwave & $1: 200$ & Becton Dickinson Biosciences \\
\hline E-cadherin & 36 & Microwave & $1: 100$ & Becton Dickinson Biosciences \\
\hline CEA & CEM010 & Microwave & $1: 200$ & Mochida, Takara Bio, Otsu, Japan \\
\hline CD56 & NCC-Lu-243 & Microwave & $1: 25$ & Nippon Kayaku, Tokyo, Japan \\
\hline CD29 & $7 F 10$ & Microwave & $1: 20$ & Novocastra \\
\hline $\mathrm{CD} 44$ & DF1485 & Microwave & $1: 40$ & Novocastra \\
\hline \multicolumn{5}{|c|}{ Cluster differential markers } \\
\hline CD5 & SP19 & Microwave & Prediluted & Ventana Medical Systems \\
\hline CD10 & $56 \mathrm{C} 6$ & Microwave & $1: 20$ & Dako Cytomation \\
\hline CD15 & BY87 & Microwave & Prediluted & Ventana Medical Systems \\
\hline CD20 & L26 & Microwave & Prediluted & Ventana Medical Systems \\
\hline CD30 & Ber-H2 & Microwave & Prediluted & Ventana Medical Systems \\
\hline CD133 & AC133 & Microwave & 1200 & Miltenyi Biotec $\mathrm{GmbH}$, \\
\hline & & & & Bergisch-Gladbach, Germany \\
\hline \multicolumn{5}{|l|}{ Mucin-related proteins } \\
\hline Muc-1 & Ma695 & Microwave & $1: 100$ & Novocastra \\
\hline Muc-2 & Ccp58 & Microwave & $1: 100$ & Novocastra \\
\hline Muc-5 & AC CLH2 & Microwave & $1: 50$ & Novocastra \\
\hline Muc-6 & CLH5 & Microwave & $1: 50$ & Novocastra \\
\hline M-CCMC-1 & HIK1083 & Microwave & $1: 10$ & Kanto Chemical, Tokyo, Japan \\
\hline \multicolumn{5}{|c|}{ Mesothelium-associated markers } \\
\hline $\mathrm{D} 2-40$ & $\mathrm{D} 2-40$ & Microwave & $1: 100$ & Signet laboratories \\
\hline Calretinin & Polyclonal & Microwave & $1: 100$ & Zymed \\
\hline \multicolumn{5}{|l|}{ Hypoxic markers } \\
\hline GULT-1 & Polyclonal & Microwave & $1: 200$ & Thermo Fisher Scientific, CA, USA \\
\hline CA-IX & Polyclonal & Microwave & $1: 150$ & Santa Cruz, Santa Cruz, CA, USA \\
\hline \multicolumn{5}{|l|}{ Extracellular matrix } \\
\hline Laminin-5 & P3H9-2 & Microwave & $1: 100$ & R\&D System \\
\hline
\end{tabular}


Table 2 The average of staining scores in thymomas and thymic carcinoma

\begin{tabular}{|c|c|c|c|c|}
\hline \multicolumn{5}{|c|}{ Histology } \\
\hline Antibodies & $A$ & $B 1,2$ & B3 & Carcinoma \\
\hline \multicolumn{5}{|l|}{ Cytokeratins } \\
\hline CK1 & $0 \pm 00$ & $0 \pm 00$ & $1 \pm 01$ & $1 \pm 01$ \\
\hline CK4 & $55 \pm 09$ & $57 \pm 12$ & $116 \pm 14$ & $105 \pm 12$ \\
\hline CK5/6 & $233 \pm 20$ & $156 \pm 26$ & $248 \pm 20$ & $250 \pm 22$ \\
\hline CK7 & $123 \pm 35$ & $33 \pm 18$ & $73 \pm 27$ & $45 \pm 19$ \\
\hline CK8 & $105 \pm 28$ & $113 \pm 28$ & $108 \pm 37$ & $94 \pm 19$ \\
\hline CK10 & $0 \pm 00$ & $0 \pm 00$ & $0 \pm 00$ & $0 \pm 00$ \\
\hline CK13 & $17 \pm 08$ & $5 \pm 05$ & $109 \pm 27$ & $52 \pm 16$ \\
\hline CK14 & $60 \pm 20$ & $14 \pm 09$ & $85 \pm 44$ & $63 \pm 15$ \\
\hline CK15 & $1 \pm 01$ & $3 \pm 02$ & $122 \pm 42$ & $44 \pm 15$ \\
\hline CK17 & $84 \pm 27$ & $50 \pm 13$ & $50 \pm 18$ & $137 \pm 24$ \\
\hline CK18 & $20 \pm 05$ & $0 \pm 00$ & $42 \pm 23$ & $110 \pm 19$ \\
\hline CK19 & $232 \pm 31$ & $249 \pm 21$ & $231 \pm 28$ & $180 \pm 27$ \\
\hline CK20 & $1 \pm 01$ & $0 \pm 00$ & $0 \pm 00$ & $0 \pm 00$ \\
\hline \multicolumn{5}{|c|}{ Cluster differential markers } \\
\hline CD5 & $0 \pm 00$ & $0 \pm 00$ & $0 \pm 00$ & $33 \pm 18$ \\
\hline CD10 & $6 \pm 04$ & $3 \pm 03$ & $0 \pm 00$ & $42 \pm 29$ \\
\hline CD15 & $157 \pm 46$ & $13 \pm 08$ & $0 \pm 00$ & $45 \pm 21$ \\
\hline CD20 & $12 \pm 10$ & $13 \pm 09$ & $0 \pm 00$ & $0 \pm 00$ \\
\hline CD30 & $0 \pm 00$ & $0 \pm 00$ & $0 \pm 00$ & $0 \pm 00$ \\
\hline CD117 (c-kit) & $2 \pm 00$ & $8 \pm 00$ & $44 \pm 00$ & $162 \pm 00$ \\
\hline CD133 & $0 \pm 00$ & $0 \pm 00$ & $0 \pm 00$ & $0 \pm 00$ \\
\hline \multicolumn{5}{|c|}{ Adhesion molecules } \\
\hline$\beta$-Catenin & $221 \pm 21$ & $169 \pm 16$ & $150 \pm 15$ & $179 \pm 19$ \\
\hline E-cadherin & $224 \pm 20$ & $111 \pm 29$ & $159 \pm 23$ & $253 \pm 18$ \\
\hline CD56 & $2 \pm 01$ & $0 \pm 00$ & $0 \pm 00$ & $10 \pm 07$ \\
\hline CD29 & $290 \pm 07$ & $255 \pm 19$ & $198 \pm 25$ & $233 \pm 21$ \\
\hline CD44 & $34 \pm 05$ & $103 \pm 16$ & $88 \pm 16$ & $150 \pm 33$ \\
\hline CEA & $0 \pm 00$ & $0 \pm 00$ & $0 \pm 00$ & $42 \pm 25$ \\
\hline \multicolumn{5}{|c|}{ Cytoskeletal-associated markers } \\
\hline S-100 & $3 \pm 03$ & $13 \pm 05$ & $11 \pm 04$ & $28 \pm 12$ \\
\hline Vimentin & $52 \pm 29$ & $117 \pm 19$ & $34 \pm 21$ & $30 \pm 17$ \\
\hline \multicolumn{5}{|c|}{ Apoptosis-associated proteins } \\
\hline bcl-2 & $162 \pm 24$ & $5 \pm 05$ & $1 \pm 01$ & $192 \pm 36$ \\
\hline P53 & $39 \pm 16$ & $11 \pm 04$ & $44 \pm 20$ & $36 \pm 12$ \\
\hline P63 & $212 \pm 22$ & $117 \pm 26$ & $220 \pm 21$ & $237 \pm 18$ \\
\hline \multicolumn{5}{|c|}{ Growth factors and hormone receptors } \\
\hline EGFR & $124 \pm 26$ & $86 \pm 13$ & $129 \pm 10$ & $124 \pm 19$ \\
\hline c-erbB-2 & $0 \pm 00$ & $0 \pm 00$ & $0 \pm 00$ & $0 \pm 00$ \\
\hline PDGFR $\beta$ & $0 \pm 00$ & $9 \pm 09$ & $9 \pm 09$ & $12 \pm 08$ \\
\hline IGFR & $188 \pm 36$ & $171 \pm 28$ & $223 \pm 39$ & $197 \pm 27$ \\
\hline PgR & $0 \pm 00$ & $0 \pm 00$ & $1 \pm 01$ & $0 \pm 00$ \\
\hline ER & $0 \pm 00$ & $0 \pm 00$ & $6 \pm 06$ & $0 \pm 00$ \\
\hline $\mathrm{AR}$ & $0 \pm 00$ & $18 \pm 18$ & $1 \pm 01$ & $0 \pm 00$ \\
\hline \multicolumn{5}{|c|}{ Drug-resistant gene products } \\
\hline ERCC-1 & $19 \pm 08$ & $45 \pm 24$ & $59 \pm 25$ & $29 \pm 15$ \\
\hline \multicolumn{5}{|c|}{ Mucin-related proteins } \\
\hline Muc-1 & $0 \pm 00$ & $0 \pm 00$ & $0 \pm 00$ & $54 \pm 00$ \\
\hline Muc-2 & $19 \pm 00$ & $0 \pm 00$ & $0 \pm 00$ & $0 \pm 00$ \\
\hline Muc-5 & $0 \pm 00$ & $0 \pm 00$ & $0 \pm 00$ & $8 \pm 00$ \\
\hline Muc-6 & $0 \pm 00$ & $1 \pm 00$ & $0 \pm 00$ & $3 \pm 00$ \\
\hline M-CCMC-1 & $0 \pm 00$ & $0 \pm 00$ & $0 \pm 00$ & $0 \pm 00$ \\
\hline \multicolumn{5}{|c|}{ Mesothelium-associated markers } \\
\hline Podoplanin & $2 \pm 01$ & $31 \pm 24$ & $87 \pm 29$ & $0 \pm 00$ \\
\hline Calretinin & $12 \pm 05$ & $0 \pm 00$ & $21 \pm 14$ & $60 \pm 33$ \\
\hline \multicolumn{5}{|l|}{ Hypoxic markers } \\
\hline CA-IX & $0 \pm 00$ & $0 \pm 00$ & $15 \pm 11$ & $110 \pm 30$ \\
\hline GULT-1 & $7 \pm 07$ & $0 \pm 00$ & $4 \pm 03$ & $167 \pm 23$ \\
\hline \multicolumn{5}{|c|}{ Extracellular matrix } \\
\hline Laminin-5 & $7 \pm 07$ & $0 \pm 00$ & $0 \pm 00$ & $12 \pm 07$ \\
\hline
\end{tabular}

Table 3 Staining score of type B3 thymoma and thymic carcinoma

\begin{tabular}{lcc}
\hline Antibody & B3 vs Ca & P-value \\
\hline CK18 & 42 vs 110 & 0.0460 \\
GULT-1 & 4 vs 167 & $<0.0001$ \\
CA-IX & 15 vs 110 & 0.0099 \\
c-kit & 44 vs 162 & 0.0056 \\
CD5 & 0 vs 33 & $<0.0001$ \\
MUC-1 & 0 vs 54 & $<0.0001$ \\
CEA & 0 vs 42 & $<0.0001$ \\
\end{tabular}

\section{Results}

\section{Staining Scores for the Thymic Carcinomas and Thymomas}

The thymic carcinoma and thymoma scores for staining with each antibody are summarized in Table 2. Antibodies whose staining score showed the statistical differences between thymic carcinoma and type B3 thymoma were shown in Table 3.

\section{Cytokeratins}

Of the 13 cytokeratins tested, the thymic carcinoma scores for CK18 staining were significantly higher (Figure 2a) than the thymoma type B3 scores $(P=0.0460)$. The average staining score of thymic carcinomas was 110 , as opposed to 42 for type B3 thymomas. The average staining score for CK17 was 137 in the thymic carcinomas and 50 in type B3 thymomas, and the difference was significant $(P=0.0118)$. However, the average staining score of type A, and type B1, B2 thymomas were 84 and 50; and the statistical difference between the type A thymomas and thymic carcinomas was not significant $(P=0.1627)$. No significant differences between the thymic carcinomas and type B3 thymomas in the expression of the other cytokeratins were found.

\section{Hypoxia-related markers}

The scores of the thymic carcinomas staining for glucose transporter 1 (GLUT-1) and carbonic anhydrase IX (CA-IX) were significantly higher. The GLUT-1 was unequivocally expressed in the membrane and cytoplasmic staining in the tumor cells. The GLUT-1 staining pattern of the thymic carcinomas tended to be more intensely positive in the central area (arrow) of tumor nests than in the peripheral area (arrow head) (Figure 2b, c). The average staining score for GLUT-1 was 167 in the thymic carcinomas and 4 in the type B3 thymomas, and the difference was significant $(P<0.0001)$.

The CA-IX showed membrane and cytoplasmic staining in the tumor cells. (Figure 2d). The average staining score for CA-IX was 110 in the thymic carcinomas and 15 in the type B3 thymomas, and the difference was significant $(P=0.0099)$. 


\section{Cluster differential markers}

The average staining score for c-kit (CD117) was 162 in the thymic carcinomas and 44 in the type B3 thymomas, and the difference was significant $(P<0.0056)$ (Figure 2e). The average staining score for CD5 was 33 in the thymic carcinomas (Figure 2f) and 0 in all of the types of thymomas, and the difference was statistically significant between thymic carcinomas and all types of thymomas $(P<0.0001)$.

\section{Mucin-related proteins and adhesion molecules} The average staining score for MUC-1 was 54 in the thymic carcinomas and 0 in the type B3 thymomas, and the average staining score for CEA was 42 in thymic carcinomas and 0 in the type B3 thymomas. The scores for both MUC-1 and CEA were the significantly higher in the thymic carcinomas than in all of the types of thymomas $(P<0.0001)$ (Figure $2 \mathrm{~g}, \mathrm{~h})$.

\section{Mesothelium-associated markers}

Podoplanin was mainly expressed on the membranous reaction of the tumor cells in the type B3 thymomas and in one case of type B2 thymoma. The average staining score for podoplanin was 87 in the type B3 thymomas, whereas the staining score was 0 in every case of thymic carcinoma, and the difference was significant $(P<0.0001)$. The average staining score for calretinin was 60 in the thymic carcinomas and 21 in type B3 thymoma, but the difference was not significant.

\section{Other antibodies}

We evaluated the expression of 42 other biological markers, but no differences in expression were found between the thymic carcinomas and type B3 thymomas.

\section{Sensitivity and Specificity of Positive Immunostaining for GLUT-1, CA-IX, c-kit, CD5, CK18, MUC-1, and CEA for Thymic Carcinoma}

We then evaluated the sensitivity and specificity of positive immunostaining for these seven markers in thymic carcinoma, and the results are shown in Table 4. GLUT-1, CD5, MUC-1, and CEA showed $100 \%$ specificity for thymic carcinoma, and GLUT1, CA-IX, c-kit, and CK18 displayed higher sensitivity. The GLUT-1 showed the highest sensitivity and specificity in these seven markers.
We selected the four markers whose positive immunohistochemical staining showed $100 \%$ specificity for thymic carcinoma: GLUT-1, CD5, MUC-1, and CEA, and validated their immunoreactivity in 12 cases of the thymic carcinoma (Table 5). A total of 8 of the 12 cases of thymic carcinoma were GLUT-1 positive, and 11 cases were positive for GLUT-1, CD5, and/or CEA. Case no. 9 did not show reactivity for any markers. Immunostaining of a combination of three markers, GLUT-1, CD5, and CEA, yielded $91.6 \%$ sensitivity and $100 \%$ specificity for thymic carcinoma.

\section{Discussion}

The results of this study showed that the seven markers selected, that is, GLUT-1, CA-IX, c-kit, CD5, MUC-1, CEA, and CK18, were useful for differentiating between type B3 thymoma and thymic carcinoma. Two of these markers, c-kit and CD5, have already been shown in many publications., ${ }^{4,3,7,5,9,8}$ Among these 'positive' thymic carcinoma markers identified in the current study, GLUT-1 may be one of the best markers for diagnosing thymic carcinoma for the following reasons. First, GLUT-1 is the marker, which showed the highest sensitivity and specificity for thymic carcinoma (Table 4). The results of this study showed that $72.7 \%$ of the thymic carcinoma stained positive for GLUT-1 as opposed to $0 \%$ of the type B3 thymoma. The staining pattern in thymic carcinoma tended to more intense in the central area of the tumor nests than in the peripheral area (Figure $2 b$ ). The average staining score for GLUT-1 (167 \pm 23$)$ was high, and much higher than that for CD5 $(33 \pm 18)$, which is a well-known thymic carcinoma marker.

Glucose transporter 1 is thought to be a possible intrinsic marker of hypoxia, and the expression of GLUT-1 has been found to be regulated by hypoxia in a HIF-1-dependent way. ${ }^{16,17}$

Glucose transporter 1 is one of 14 members of the mammalian facilitative glucose transporter (GLUT) family of passive carriers that function as an energyindependent system for transport of glucose down a concentration gradient. ${ }^{18,19}$ GLUT-1 is expressed in a variety of carcinomas, including carcinoma of the breast, head and neck, ovary, renal cell carcinoma, lung, and malignant pleural mesothelioma. ${ }^{20-26}$ Positron emission tomography (PET) imaging using [18F]2-fluoro-2-deoxy-D-glucose (FDG) has

Figure 2 Results of immunostaining for CK18, GLUT-1, CA-IX, c-kit, CD5, MUC-1, and CEA in thymic carcinoma. (a) CK18 immunostaining in a thymic carcinoma with a staining score of 120 (positive cells, 60\%; staining intensity, 2+). (b) and (c) GLUT-1 immunostaining in a thymic carcinoma with a staining score of 210 (positive cells, $70 \%$; staining intensity, 3+). (d) CA-IX immunostaining in a thymic carcinoma with a staining score of 120 (positive cells, 60\%; staining intensity, 2+). (e) c-kit immunostaining in a thymic carcinoma with a staining score of 210 (positive cells, $70 \%$; staining intensity, $3+$ ). (f) CD5 immunostaining in a thymic carcinoma with a staining score of 80 (positive cells, 40\%; staining intensity, $2+$ ). (g) MUC-1 immunostaining in a thymic carcinoma with a staining score of 60 (positive cells, 30\%; staining intensity, $2+$ ), and (h) CEA immunostaining in a thymic carcinoma with a staining score of 60 (positive cells, $30 \%$; staining intensity, $2+$ ). 
the potential value of delineating viable tumor tissue on the basis of increased glucose metabolism with the tumor. ${ }^{27-30}$ Sasaki et $a l^{31}$ found that FDG-
PET can be used to differentiate benign thymic tumors from thymic carcinoma. In view of previous reports of a correlation between the FDG-PET
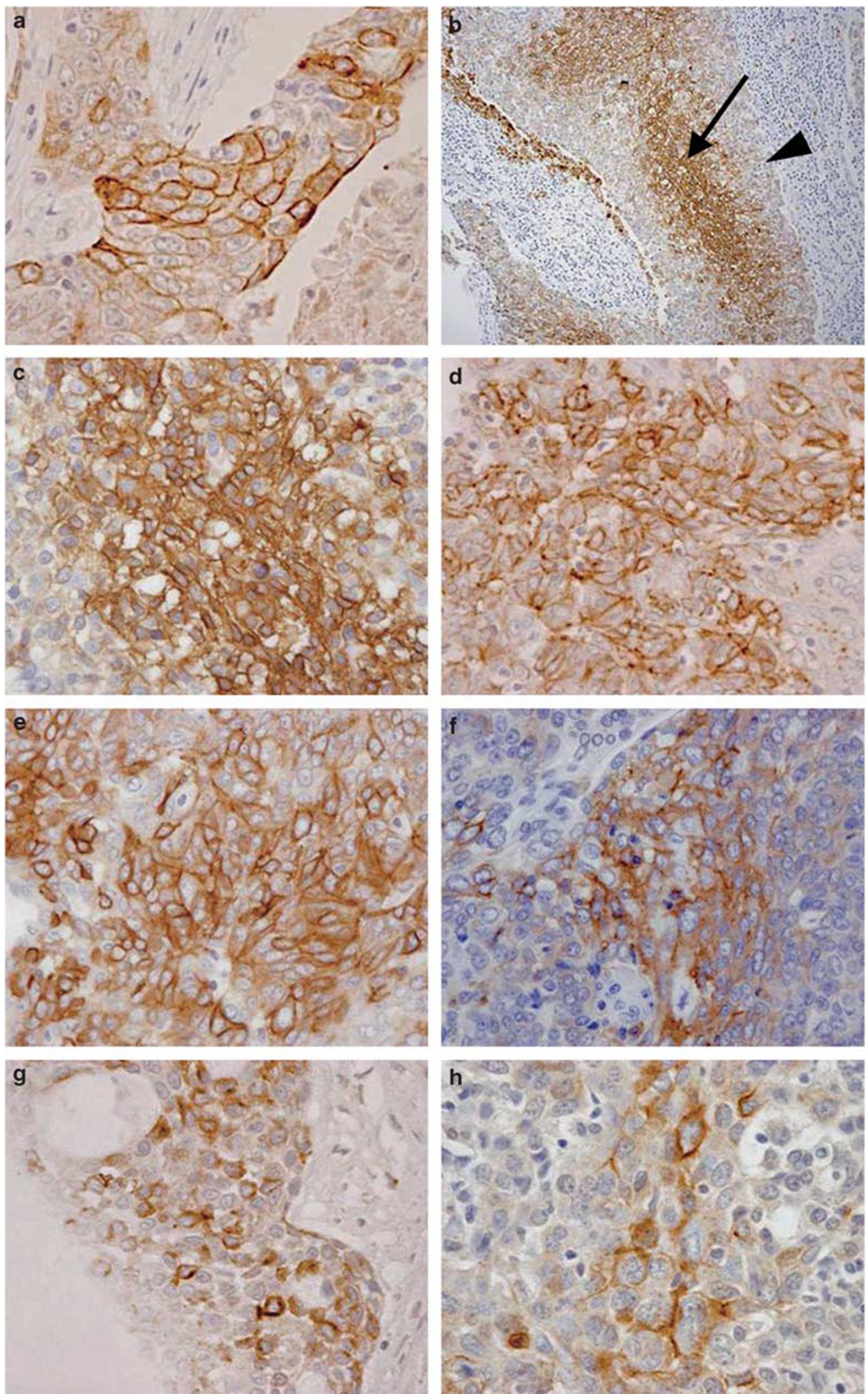
Table 4 Sensitivity and specificity for thymic carcinoma

\begin{tabular}{lcc}
\hline Antibody & Sensitivity (positive ratio) (\%) & Specificity (\%) \\
\hline CK18 & 58 & 71 \\
GLUT-1 & 72 & 100 \\
CA-IX & 58 & 71 \\
c-kit & 72 & 85 \\
CD5 & 33 & 100 \\
MUC-1 & 33 & 100 \\
CEA & 25 & 100 \\
\hline
\end{tabular}

Table 5 Immunostaining result of GLUT-1, CD5, CEA, and MUC-1 in each thymic carcinoma case

\begin{tabular}{lccccc}
\hline \multicolumn{7}{c}{ Antibody } \\
\hline Case & GLUT-1 & CD5 & CEA & MUC-1 & $\begin{array}{c}\text { Combination of } \\
\text { three markers }\end{array}$ \\
& & & & & + \\
\hline 1 & - & + & - & - & + \\
2 & + & - & - & - & + \\
3 & + & - & - & + & + \\
4 & + & - & - & - & + \\
5 & + & + & - & - & + \\
6 & - & - & + & - & + \\
7 & + & + & + & - & + \\
8 & + & - & - & + & + \\
9 & - & - & - & - & + \\
10 & NA & + & - & + & + \\
11 & + & - & - & - & + \\
12 & + & - & + & + & + \\
& + & & & & +
\end{tabular}

NA, not available.

${ }^{\mathrm{a}}$ Combination of three markers: GLUT-1 and/or CD5 and/or CEA.

findings and GLUT-1 staining, our findings might be consistent with the results of the immunohistochemical staining in the present study.

Immunostaining for CA-IX, ${ }^{16,32}$ another hypoxic marker and a major downstream target of HIF-1 $\alpha$, was also significantly stronger in the thymic carcinomas (staining score: 110), the same as immunostaining for GLUT-1, but its specificity was lower $(58 \%$ of thymic carcinomas and $28 \%$ of type B3 thymomas stained positive for CA-IX).

The fact that the CA-IX score in other types of thymomas was 0 , suggests that a hypoxic microenvironment is a specific characteristic of thymic carcinomas as compared with thymomas.

MUC-1 and CEA showed 100\% sensitivity for thymic carcinoma. Both markers might be suitable for making the differential diagnosis, but only 33.3 and $25.0 \%$, respectively, of the thymic carcinoma stained positive and the staining scores were lower than for GLUT-1. In view of these low staining scores, these markers alone may not be suitable for use in small biopsy specimens, such as those obtained by needle biopsy.

Previous studies reported CD5-positive rates of $50-100 \%,,^{9,8,11,33}$ however, our study shows the lower CD5-positive rate (33\%). This would be explained by a smaller tissue of tissue microarray. c-kit (CD117) has been reported to be a useful marker for the diagnosis of thymic carcinoma., ${ }^{5,9}$ Previous studies reported positive immunoreactivity for c-kit in $65-91 \%$ of thymic carcinomas, as opposed to $0-5 \%$ of thymoma. ${ }^{5,9,32,34}$ In our study, staining for c-kit was only focally weakly positive in 2 of the 7 cases of type B3 thymoma, and was more extensively expressed in 8 of the 11 cases of thymic carcinoma.

The staining score for CK18 was significantly higher in the thymic carcinomas, consistent with a previous report. ${ }^{35}$ Fukai et al showed that CK18 was expressed to a greater extent on the epithelium of thymic carcinomas than on the epithelium of thymomas, including types $\mathrm{A}, \mathrm{B} 1, \mathrm{~B} 2$, and $\mathrm{AB}$. In their study, although thymic carcinoma shows $33 \%$ of diffuse-positive staining reaction of tumor cells with CK18, but none of the types of thymoma show the diffuse reaction. They suggested that the CK18positive epithelial cells in thymomas may transform into thymic carcinoma cells under certain conditions.

Podoplanin is expressed in normal tissue, including lymphatic endothelium and mesothelium, and in neoplasms, including pulmonary squamous cell carcinomas and malignant mesothelioma. ${ }^{36,37}$ In this study, $57.1 \%$ of the type B3 thymomas stained positive for podoplanin, whereas none of the thymic carcinomas reacted with the antibody. This finding is very interesting, and an analysis after accumulation of additional cases is necessary.

When it is difficult to make the differential diagnosis with a single antibody, it is helpful to use a combination of antibodies. ${ }^{10,38}$ In this study, combined use of the three antibodies that yielded $100 \%$ specificity for thymic carcinoma, that is, the antibodies for GLUT-1, CD5, and CEA, provided $91.6 \%$ sensitivity. Thus, combined use of these three antibodies should be useful for differentiating between thymic carcinoma and type B3 thymoma in biopsy specimens that have been crushed or are otherwise difficult to examine morphologically.

In conclusion, we found that immunostaining for GLUT-1, CA-IX, c-kit, CD5, CK18, MUC-1, and CEA enables differential diagnosis between thymic carcinoma and thymoma type B3. The combination of positive immunostaining for GLUT-1, CD5, and CEA yielded $91.6 \%$ sensitivity and $100 \%$ specificity for thymic carcinoma. A prospective study of a larger number of cases is required to define the expression of these molecules more precisely in order to enable pathologists to reliably differentiate between thymic carcinoma and type B3 thymoma.

\section{Disclosure/conflict of interest}

The authors declare no conflict of interest. 


\section{References}

1 Muller-Hermelink HK, Engel P, Kuo TT, et al. Tumours of the thymus. In: Travis WD, Brambilla E, MüllerHermelink HK et al (eds). Pathology \& Genetics, Tumours of the Lung, Pleura, Thymus and Heart. IARC Press: Lyon, France, 2004, pp 145-247.

2 Rosai J. Histological Typing of Tumours of the Thymus. Springer-Verlag: Berlin, 1999.

3 Berezowski K, Grimes MM, Gal A, et al. CD5 immunoreactivity of epithelial cells in thymic carcinoma and CASTLE using paraffin-embedded tissue. Am J Clin Pathol 1996;106:483-486.

4 Hishima T, Fukayama M, Fujisawa M, et al. CD5 expression in thymic carcinoma. Am J Pathol 1994;145:268-275.

5 Pan CC, Chen PC, Chiang H. KIT (CD117) is frequently overexpressed in thymic carcinomas but is absent in thymomas. J Pathol 2004;202:375-381.

6 Shimosato Y, Kameya T, Nagaki K. Squamous cell carcinoma of the thymus. An analysis of eight cases. Am J Surg Pathol 1977;1:109-121.

7 Tateyama H, Eimoto T, Tada T, et al. Immunoreactivity of a new CD5 antibody with normal epithelium and malignant tumors including thymic carcinoma. Am J Clin Pathol 1999;111:235-240.

8 Kornstein MJ, Rosai J. CD5 labeling of thymic carcinomas and other nonlymphoid neoplasms. Am J Clin Pathol 1998;109:722-726.

9 Nakagawa K, Matsuno Y, Kunitoh H, et al. Immunohistochemical KIT (CD117) expression in thymic epithelial tumors. Chest 2005;128:140-144.

10 Ordóñez NG. What are the current best immunohistochemical markers for the diagnosis of epithelioid mesothelioma? A review and update. Hum Pathol 2007;38:1-16.

11 Pomplun S, Wotherspoon AC, Shah G, et al. Immunohistochemical markers in the differentiation of thymic and pulmonary neoplasms. Histopathology 2002;40: 152-158.

12 Tsuta K, Ishii G, Nitadori J, et al. Comparison of the immunophenotypes of signet-ring cell carcinoma, solid adenocarcinoma with mucin production, and mucinous bronchioloalveolar carcinoma of the lung characterized by the presence of cytoplasmic mucin. J Pathol 2006;209:78-87.

13 Nitadori J, Ishii G, Tsuta K, et al. Immunohistochemical differential diagnosis between large cell neuroendocrine carcinoma and small cell carcinoma by tissue microarray analysis with a large antibody panel. Am J Clin Pathol 2006;125:682-692.

14 Saijo T, Ishii G, Nagai K, et al. Differences in clinicopathological and biological features between central-type and peripheral-type squamous cell carcinoma of the lung. Lung Cancer 2006;52:37-45.

15 Honda T, Tamura G, Endoh Y, et al. Expression of tumor suppressor and tumor-related proteins in differentiated carcinoma, undifferentiated carcinoma with tubular component and pure undifferentiated carcinoma of the stomach. Jpn J Clin Oncol 2005;35: 580-586.

16 Vleugel MM, Greijer AE, Shvarts A, et al. Differential prognostic impact of hypoxia induced and diffuse HIF1alpha expression in invasive breast cancer. J Clin Pathol 2005;58:172-177.

17 Elson DA, Ryan HE, Snow JW, et al. Coordinate upregulation of hypoxia inducible factor (HIF)-1a and
HIF-1 target genes during multi-stage epidermal carcinogenesis and wound healing1. Cancer Res 2000;60:6189-6195.

18 Olson AL, Pessin JE. Structure, function, and regulation of the mammalian facilitative glucose transporter gene family. Annu Rev Nutr 1996;16: 235-256.

19 Clavo AC, Brown RS, Wahl RL, et al. Fluorodeoxyglucose uptake in human cancer cell lines is increased by hypoxia. J Nucl Med 1995;36:1625-1632.

20 Younes M, Lechago LV, Somoano JR, et al. Wide expression of the human erythrocyte glucose transporter Glut1 in human cancers. Cancer Res 1996; 56:1164-1167.

21 Brown RS, Wahl RL. Overexpression of Glut-1 glucose transporter in human breast cancer. An immunohistochemical study. Cancer 1993;72:2979-2985.

22 Mellanen P, Minn H, Grenman R, et al. Expression of glucose transporters in head-and-neck tumors. Int J Cancer 1994;56:622-629.

23 Nagase Y, Takata K, Moriyama N, et al. Immunohistochemical localization of glucose transporters in human renal cell carcinoma. J Urol 1995;153: 798-801.

24 Younes M, Brown RW, Stephenson M, et al. Overexpression of Glut1 and Glut3 in stage I nonsmall cell lung carcinoma is associated with poor survival. Cancer 1997;80:1046-1051.

25 Rudlowski C, Moser M, Becker AJ, et al. GLUT1 mRNA and protein expression in ovarian borderline tumors and cancer. Oncology 2004;66:404-410.

26 Kato Y, Tsuta K, Seki1 K, et al. Immunohistochemical detection of GLUT-1 can discriminate between reactive mesothelium and malignant mesothelioma. Mod Pathol 2007;20:215-220.

27 Higashi K, Nishikawa T, Oguchi M, et al. Comparison of fluorine-18-FDG PET and thallium-201 SPECT in evaluation of lung cancer. J Nucl Med 1998;39:9-15.

28 Brown RS, Leung JY, Kison PV, et al. Glucose transporters and FDG uptake in untreated primary human non-small cell lung cancer. J Nucl Med 1999;40:556-565.

29 El-Bawab H, Al-Sugair AA, Rafay M, et al. Role of flourine-18 fluorodeoxyglucose positron emission tomography in thymic pathology. Eur J CardioThorac Surg 2007;31:731-736.

30 Yasuda S, Ide M, Fujii H, et al. Application of positron emission tomography imaging to cancer screening. Br J Cancer 2000;12:1607-1611.

31 Sasaki M, Kuwabara Y, Ichiya Y, et al. Differential diagnosis of thymic tumors using a combination of 11C-methionine PET and FDG PET. J Nucl Med 1999;40:1595-1601.

32 Kon-no H, Ishii G, Nagai K, et al. Carbonic anhydrase IX expression is associated with tumor progression and a poor prognosis of lung adenocarcinoma. Lung Cancer 2006;54:409-418.

33 Nonaka D, Henley JD, Chiriboga L, et al. Diagnostic utility of thymic epithelial markers CD205 (DEC205) and Foxn1 in thymic epithelial neoplasms. Am J Surg Pathol 2007;31:1038-1044.

34 Barisella M, Andreola S, Rosai J. CD117 in soft tissue sarcomas. Am J Clin Pathol 2002;118: 470-471.

35 Fukai I, Masaoka A, Hashimoto T, et al. Cytokeratins in normal thymus and thymic epithelial tumors. Cancer 1993;71:99-105. 
36 Ordóñez NG. D2-40 and podoplanin are highly specific and sensitive immunohistochemical markers of epithelioid malignant mesothelioma. Hum Pathol 2005; 36:372-380.

37 Ordóñez NG. The diagnostic utility of immunohistochemistry in distinguishing between epithelioid me- sotheliomas and squamous carcinomas of the lung: a comparative study. Mod Pathol 2006;19:417-428.

38 Kushitani K, Takeshima Y, Amatya VJ, et al. Immunohistochemical marker panels for distinguishing between epithelioid mesothelioma and lung adenocarcinoma. Pathol Int 2007;57:190-199. 